\title{
Wall conditioning in ASDEX Upgrade
}

\author{
V. Rohde* a, R. Dux ${ }^{\mathrm{a}}$, A. Kallenbach ${ }^{\mathrm{a}}, \mathrm{K} . \mathrm{Krieger}^{\mathrm{a}}, \mathrm{R} . \mathrm{Neu}^{\mathrm{a}}$, \\ ASDEX Upgrade Team, \\ a Max-Planck-Institut für Plasmaphysik, EURATOM Association, Boltzmannstr. 2, \\ D-85748 Garching, Germany
}

\begin{abstract}
An overview on wall conditioning in ASDEX Upgrade is presented. Helium glow discharges (HeGD) are needed mostly for plasma start up after high density discharges, disruptions and disruption mitigation gas puffs. Boronisation is routinely applied. The reduction of the oxygen content is a minor effect. Strong variation of the wall pumping is observed for tungsten first wall materials. The uncoated tungsten surface stores and releases large amounts of He, which can disturb the plasma. The released He causes the modification in the wall pumping. By reducing HeGD this effect could be minimized. Advanced and natural density scenarios are sensitive to the status of the wall coating. Accumulation of impurities at the pedestal influences the ELM frequency and finally causes radiation unstable discharges.
\end{abstract}

PACS: $52.40 \mathrm{Hf}, 52.25 \mathrm{Vy}, 52.25 \mathrm{Xz}, 81.15 \mathrm{Cf}$

JNM keywords:First Wall Materials, Plasma Material Interaction, Impurities PSI-17 Keywords: ASDEX Upgrade, Boronisation, Gas injection and fueling, wall conditiong, wall pumping, 
* Corresponding author address: Max-Planck Institut für Plasmaphysik, Boltzmannstr. 2, 85478 Garching, Germany

* Corresponding author e-mail: Volker.Rohde@ipp.mpg.de presenting author: Volker Rohde presenting author e-mail:Volker.Rohde@ipp.mpg.de

\section{Introduction}

For present day devices, wall conditioning is required to reach high plasma performance. Obviously the techniques used today, which rely on thin layers, would be not sufficient for a next step device such as ITER. To get an estimate on the methods required, an understanding of the mechanism involved in present day devices is required. In ASDEX Upgrade (AUG) a step by step replacement of carbon plasma facing components by tungsten coated ones was started, which will finally lead to a carbon free divertor tokamak. During this transition the influence of wall conditioning on the plasma performance, especial for low density scenarios, becomes more and more important.

The aim of wall conditioning is to optimize the influence of the plasma facing components on the plasma performance. An unconditioned wall releases gaseous species, which can prevent stable plasma operation, whereas a pumping wall is favoured. To minimize the gas release one has first to reduce the wall inventory. In this sense a leak check, to reduce the external leakage, is part of the wall conditioning. For experimental operation a base pressure of $1 x 10^{-5} \mathrm{~Pa}$ without operating the in-vessel cryo pump is reached. Further reductions of the external leakage require disproportional effort. Due to the 
amount of graphite (approx. $1000 \mathrm{~kg}$ ) built into the vessel internal leakage dominated. Limited baking temperatures of $150{ }^{\circ} \mathrm{C}$ require $72 \mathrm{~h}$ baking to reduce the water partial pressure. After the baking the residual gas consists of an equal amount of air and water. Further reduction of the base pressure reached by to plasma operation. After the baking, wall coating is performed to reduce the oxygen content. It had been shown that plasma operation without wall coating is possible, but the plasma performance was not satisfactory.

\section{Glow discharge cleaning}

Helium glow discharges (HeGDs) are routinely used in-between shots. The purpose is to clean the wall from impurities and working gas. Typical e-fold decay times of $300 \mathrm{~s}$ for $D_{2}$ and $100 \mathrm{~s}$ for hydro-carbons are measured by residual gas analysis. HeGDs enable plasma start up after disruptions and high density discharges. Additionally the disruption mitigation puffs, which are used routinely, inject huge amounts of Ne into the vessel [1]. This noble gas has to be removed from the wall to be pumped out of the torus. The operation scheme had been 15 min glow on the morning of an experimental day and 5 min in-between two shots. As discussed below, high He contents had been observed during the last campaign, so the HeGD had to be restricted. The glow time had been reduced to $5 \mathrm{~min}$ and $3 \mathrm{~min}$ respectively, and it is only applied if needed. Mostly low density discharges are hindered by a

high He content. Low density discharges did not load the wall, so no glowing is needed in between shots. Combining a series of low density shots further reduces the He content. High density scenarios, which load the wall, are not hindered by the He outgasing. Additionally new techniques such as ICRF-GD 
have been developed to enable wall cleaning with magnetic field, as required in superconducting devices [2].

\section{$3 \quad$ Wall coating}

For optimal plasma conditions wall coating procedures such as boronisation or siliconisation are used [3]. Both techniques are based on HeGD with an additional inlet of $10 \%$ reactive gases like diborane. Since the used gases are toxic, a special gas inlet and pumping system is needed. The gas is fed into the torus at two positions; 4 glow anodes are used for an homogeneous glow discharge. The homogeneity of the discharges could be controlled by eye or video camera and the voltage at the anodes. Typical properties of the discharge are a controlled current of $7 \mathrm{~A}$, a voltage of $540 \mathrm{~V}$ and a pressure of $0.52 \mathrm{~Pa}$. From residual gas analysis a deposition efficiency of $97 \%$ is derived. During a boronisation typically $4.2 \times 10^{23}$ at of B have been deposited. The thickness of the deposited layer is monitored using a quartz microbalance monitor. Additionally deposition probes could be inserted using a manipulator system. From ion beam analysis of these samples the layer thickness and composition could be derived. Results are shown in table 1. From the gas inlet a layer thickness of $8.5 \times 10^{21} \mathrm{at} / \mathrm{m}^{2}$ is expected in good agreement with the measurement. This again hints to the fact, that the layers are deposited uniformly around the torus. The layer is thicker on a $\mathrm{C}$ substrate than on a Si wafer. From TRIDYN calculations it is expected that the re-erosion by the glow discharge is stronger for higher Z substrates [4]. A $50 \%$ higher energy of the reflected deuterium is expected on $\mathrm{Si}$ in comparison with $\mathrm{C}$, which causes the stronger re-erosion of the deposited layers, as observed. The period of the coatings procedures 
depends on the requirements for the shot scenario. Whereas high density high power shots require no special wall coating, advanced scenarios rely on a fresh coating. In fact problems with advanced scenarios are mostly used as criterion for a new coating. Due to the effort required for a wall coating, the number of coatings has to be restricted. The average life time for a boronisation in ASDEX Upgrade is about 200 plasma discharges, or 4 weeks of experimental operation.

\section{Oxygen concentrations}

Wall coating techniques had been invented to reduce the oxygen content of a plasma discharge by gettering. To compare the status of the coating, a standard H-mode discharge, which is performed at the beginning of each experimental day is used. The natural density phase in particular, which has no external gas puff, is very sensitive to the wall status. Typical oxygen concentrations at the start of an experimental campaign are shown in Fig. 1. For the first shots concentrations of up to $2 \%$ are measured. This value is continuously decreasing during plasma operation due to the wall release by thermal load. After the next boronisation oxygen concentrations as low as 0.15 $\%$ are observed. These concentrations rise over serval experimental days to the typical value of $0.4 \%$. Obviously oxygen is not an outstanding impurity in ASDEX Upgrade. During the transition from a carbon to tungsten first wall device the oxygen content of the discharges gets less important. During the 2005 campaign a maximum oxygen content of $0.3 \%$ was observed for the same plasma conditions. 


\section{$5 \quad$ Wall pumping / Gas release}

The amount of gas puffed varies strongly for different scenarios: whereas the wall release is the only gas input for discharges at natural density, high density H-mode discharges require about $2.5 \times 10^{22}$ at/s in ASDEX Upgrade. As one monolayer of gas on the whole plasma facing wall in ASDEX Upgrade equals about $5 \times 10^{20}$ at, no strong influence of the wall status is expected for high density H-mode discharges. To investigate time evolution of the wall pumping / release, the ohmic standard shot, which is performed as the first shot on an experimental day is used. This shot is not influenced by wall loading of proceeding shots and a database covering different status of the wall exists. Here we concentrate on the plasma build up and the natural density phases. The plasma was ramped up in a limiter configuration using the central column as a limiter. At about $0.5 \mathrm{~s}$ the divertor is formed. The amount of gas puffed is feed back controlled on the electron density. As the divertor pressure is quite low during the limiter phase and therefore exhaust by the pumps is negligible, the amount of puffed gas is a measure for the wall pumping. The gas flux used for plasma build up is shown in Fig. 2a. A boronisation is indicated by the dashed lines. A fresh coating needs up to $5 \times 10^{21}$ at of deuterium for plasma build up. After some experimental days only $1 \times 10^{19}$ at, a factor of 50 less, is needed. The natural density phase of the same discharge shows significant density minima (Fig. 2b). During this phase no gas is puffed, so the wall has to replace the pumped amount of gas. An aged coating shows constant density, i.e. strong outgasing from the wall, whereas for a fresh boronisation a significant density drop is observed. The wall pumping during the plasma build up could be estimated by: $N_{\text {Wall }}=N_{\text {Puff }} N_{\text {Pump }} N_{\text {Plas }}$ with $N_{\text {Puff }}$ the 
integral of gas puffed, $N_{\text {Pump }}$ the pumped gas calculated by the pumping speed and the neutral pressure at the pumping ducts and $N_{\text {Plas }}$ the average electron density after plasma build up times the plasma volume. Results for the plasma start up are shown in Fig. 2c. The plasma inventory after $0.5 \mathrm{~s}$ is typically $4 x 10^{20}$ at. A fresh coating releases no gas or is even pumping. The situation changes within one experimental day: now the puffing is strongly reduced the plasma is completely fed by the wall release. Further insight comes from spectroscopic investigations: for an aged coating the amount of He in the core plasma reaches up to $30 \%$. The He radiation at the central column during plasma start up was measured using the HeII $L_{\text {alpha }}$ line at $30.4 \mathrm{~nm}$ and is shown in Fig. 2d. This line presents the He influx into the plasma. For a fresh boronisation almost no He influx is observed, whereas for an aged coating a strong influx is found. As only one line of sight is available and He recycling has to be taken into account, this data is only taken as a relative measure for the He influx. The behaviour of He is quite astonishing: a boronisation requires $10 \mathrm{~h}$ He glow discharge on the evening before an experimental day, but no He influx is observed in the first shots. A hydro-boron layer, as applied by a boronisation, seems to store no He. This behaviour could be verified by laboratory experiments [5]. Whereas the storage of gas on the surface should be comparable for tungsten and carbon, the release should be higher for tungsten due to the stronger recoil of the sputtering ions on the tungsten.

Since 1999 the main chamber wall material in ASDEX Upgrade had been changed from carbon to tungsten [6]. This was done stepwise starting at the central column which is used as a start up limiter. The amount of gas used for plasma build up during this transition is shown in Fig. 3. Before the build in of tungsten at the central column, about $1 \times 10^{21}$ at of deuterium was needed for 
plasma build up. No dependence on the status of the wall coating is observed. For the 2001 campaign the central column, except the equatorial part and the NBI dumps had been coated by tungsten. During this time the amount of gas varies with the status of the boronisation. For an aged boronisation only $2 \times 10^{19}$ at of deuterium has to be puffed for plasma build up. The only difference to the previous campaigns is the wall material. For a fresh coating the tungsten is covered by hydro-boron layers: almost the same amount of gas as for a carbon wall is needed. As the boron layer is eroded and the tungsten surface can interact with the plasma, the gas release of the wall is strong enough to feed the plasma: no gas puff is needed. For the 2002/03 campaign the central column had been completely covered by new tungsten coated tiles. During the 2004/05 campaign it was observed by spectroscopy that for low density discharges He became a dominant impurity. The major input of He into the vacuum chamber is HeGD. Up to this time HeDG was routinely applied to control the wall inventory (see above), so during the 2004 campaign the use of He glowing was restricted. Additionally the plasma ramp down was changed from a limiter to a divertor configuration. The achievements of these changes could be seen for the 04/05 and 05/06 campaign. The amount of gas needed for plasma build up rises again. The He content measured by CXRS spectroscopy is decreasing at the same time. This behaviour shows that the He, which is released from the tungsten, causes the variation in the gas amount needed for plasma build up. 


\section{Surface covering}

During the last years tungsten became the dominate wall material in the main chamber of ASDEX Upgrade [6]. Due to the size of ASDEX Upgrade it is not possible to match ITER relevant core and edge conditions at the same time. Especially advanced scenarios are hindered by erosion of the tungsten wall. To enable these scenarios the tungsten has to be covered by wall coatings. As tungsten concentrations are sensitive to transport effects [6], the former carbon phase is used for comparison. The pedestal carbon concentration for the high density phase of the standard H-mode shot depends on the age of the wall coating [7]. For a fresh coating concentrations of $0.5 \%$ are measured. It rises fast within 30 shots to a value of about $1 \%$. This fast rise is in agreement for the estimated time constant for the erosion of the boron layers at the main chamber limiters. The ion flux at the central column is about a factor of 10 lower, resulting in the longer time constant of about 300 shots.

For a tungsten first wall significant differences in the global plasma behaviour are found for scenarios at the natural density, i.e. density without gas puffing. Data for the standard H-mode discharge are shown in Fig. 4. A fresh boronisation constantly radiates about $12 \%$ of the input power (Fig. 4a). For an aged boronisation the radiation starts at $20 \%$ and rises continuously up to 70

$\%$. The enhanced radiation lowers the available power flux over the pedestal and consequently the ELM frequency (Fig. 4b). For the good conditioned discharge stable frequent ELMs prevent impurities from reaching the core plasma. The low ELM frequency results in an inward impurity drift, which transports more impurities over the pedestal. Whereas for a fresh boronisation the density stays constant for $1 \mathrm{sec}$, an aged boronisation shows a density rise (Fig. 
4c). Both the density and radiation rises on a $300 \mathrm{~ms}$ timescale, indicating a slow transport of the impurities into the pedestal region. The radiation finally causes degradation of the stored energy (Fig. 4d). This is an unstable cycle resulting in grassy ELM and finally in a transition to L mode. This cycle can also be triggered by puffing of impurities.

\section{Summary}

Wall conditioning is an important technique for plasma operations. HeGDs are very effective at removing gas from the wall. This is needed for plasma start up, after high density discharges and disruptions. In case of mitigated disruption the puffed noble gas has to be removed using a HeGD. After boronisation oxygen is not an outstanding impurity. For a tungsten wall strong variation of the wall release is observed. Tungsten coatings can store and release high amounts of noble gases, which cause the changes in the gas balance. Low density discharges are influenced by the released He. Intelligent use of HeGD solves this problem. Wall coating in ASDEX Upgrade is mostly needed for low density and scenarios with low auxiliar heating power. Advanced scenarios are quite sensitive on wall conditioning. Due to the good particle confinement, higher impurity concentrations are found in the core plasma. Midsize devices such as ASDEX Upgrade require a thin hot SOL for these scenarios. These conditions are not favourable for a tungsten wall. A covering of the tungsten source helps for a restricted number of discharges. 


\section{References}

[1] G. Pautasso, Fusion Science and Technology, 44 (2003), 716.

[2] A. Lyssoivan, these Proceedings, P2-72.

[3] V. Rohde, Europhys. Conf. Abstr., 23J (1999) 1513.

[4] H. Maier et al., J. Nucl. Mat. 337-339 (2005) 480.

[5] K. Schmid, to be published.

[6] R. Neu, these Proceedings, I-9.

[7] A. Kallenbach,these Proceedings, O-1. 


\section{Table 1:}

\begin{tabular}{c|ccc} 
boronisation & 2005 & 2005 & 1999 \\
wafer & $\mathrm{Si}$ & $\mathrm{C}$ & $\mathrm{Si}$ \\
\hline $\mathrm{D}+\mathrm{H}$ & $3.45 \times 10^{21} \mathrm{at} / \mathrm{m}^{2}$ & $4.11 \times 10^{21} \mathrm{at} / \mathrm{m}^{2}$ & $4.00 \times 10^{21} \mathrm{at} / \mathrm{m}^{2}$ \\
$\mathrm{C}$ & $8.4 \times 10^{20} \mathrm{at} / \mathrm{m}^{2}$ & - & - \\
& & & \\
$\mathrm{B}$ & $7.95 \times 10^{21} \mathrm{at} / \mathrm{m}^{2}$ & $1.2 \times 10^{22} \mathrm{at} / \mathrm{m}^{2}$ & $6.40 \times 10^{21} \mathrm{at} / \mathrm{m}^{2}$
\end{tabular}

Table 1: Analysis of the layers deposited during a boronisation.

Fig. 1: Oxygen concentrations for a natural density H-mode discharge. Boronisations are indicated as dashed lines.

Fig. 2 : Amount of Gas needed for plasma build up (a), natural density (b), amount for gas pumped by the wall during plasma build up (c) and He II influx (d) after 3 boronisation (dashed lines).

Fig. 3: Evolution of the amount of gas needed for plasma build up since 1998.

Fig. 4: Natural density phase of an H-mode discharge with fresh (dark) and aged (light) boronisation. Enhanced pedestal radiation (a) causes irregular ELMs (b), leading to a rising electron density (c) and finally an unstable discharge (d). 


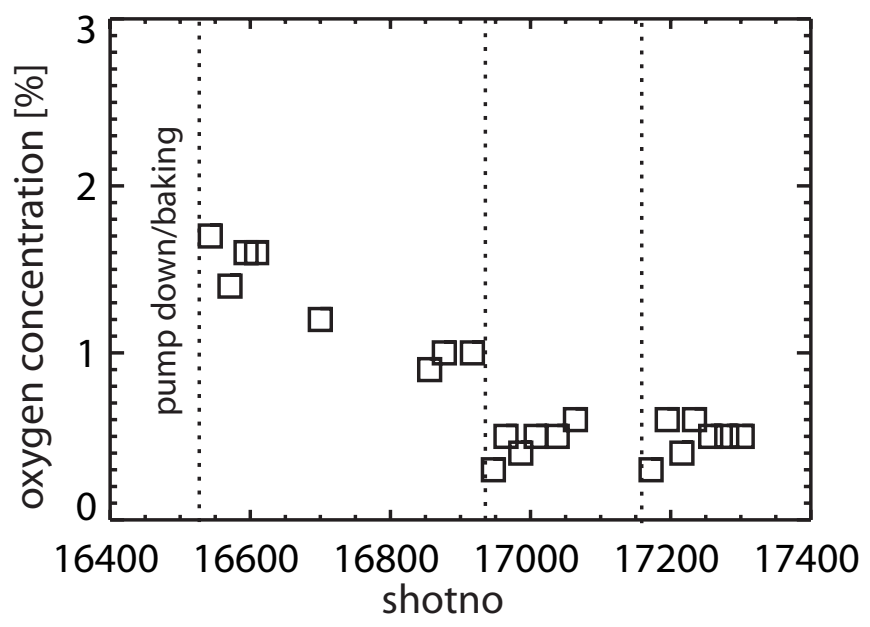

PSI17- P2-74 


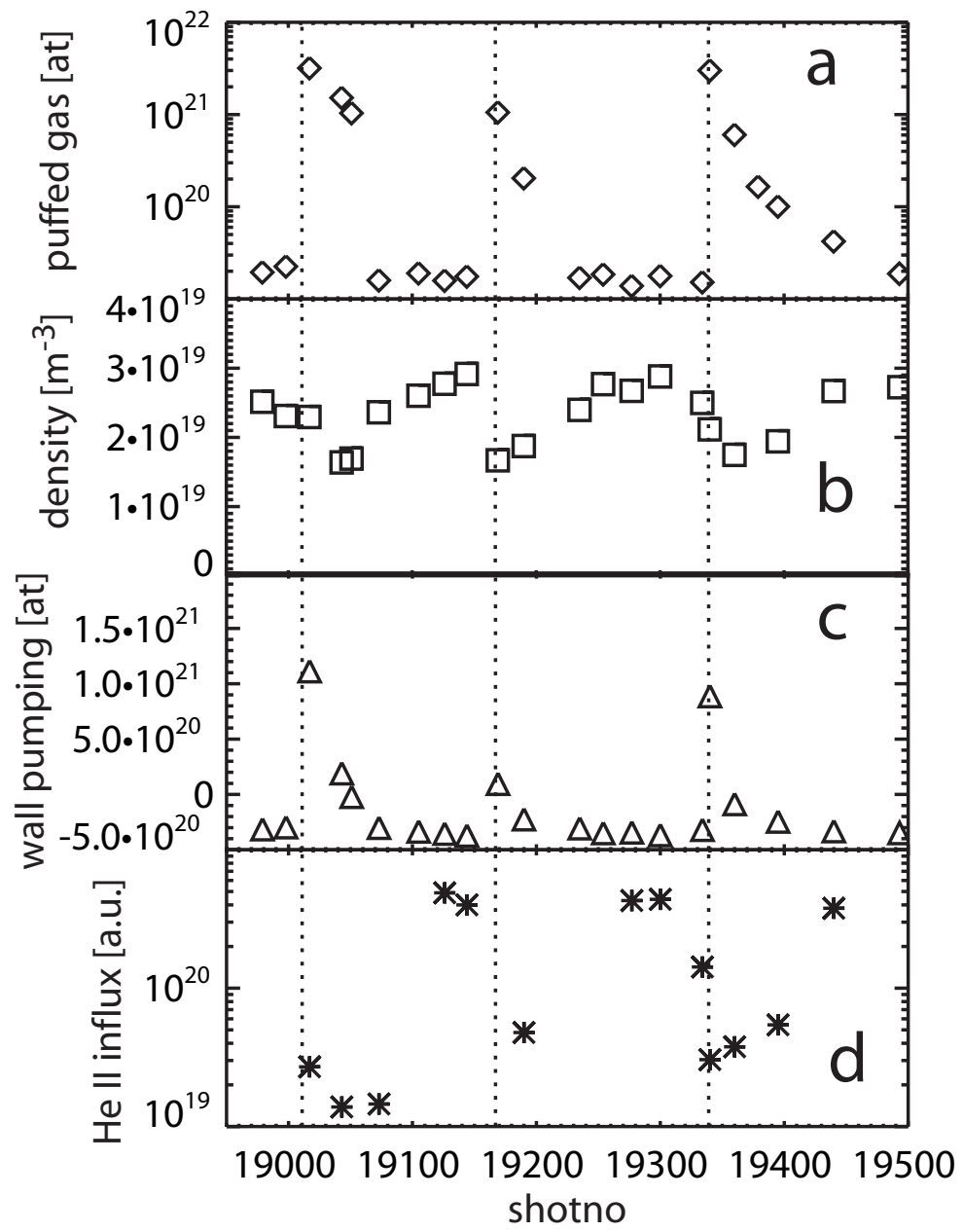

PSI17-P2-74

Fig2 


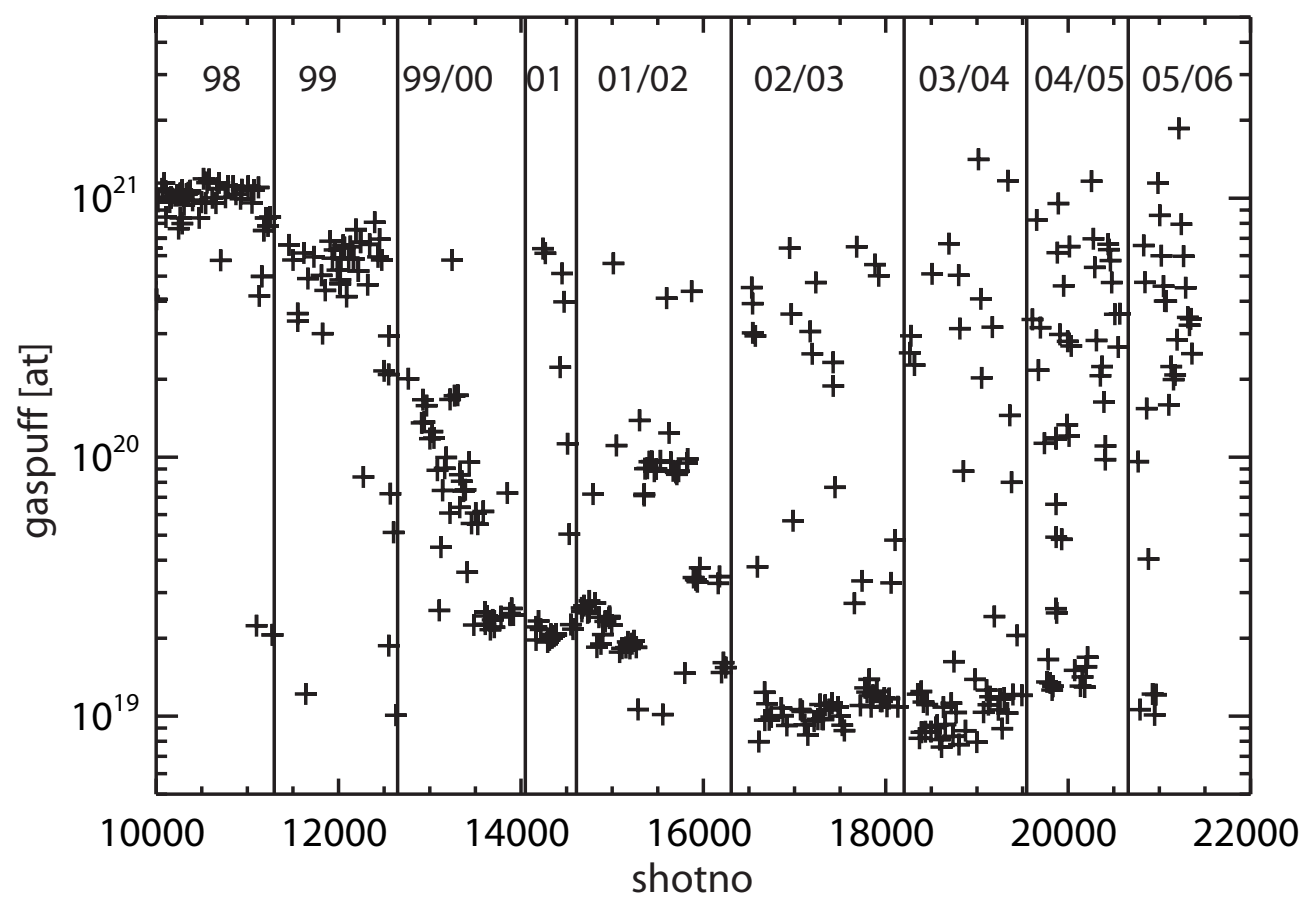

Fig 3

PSI17-P2-74 


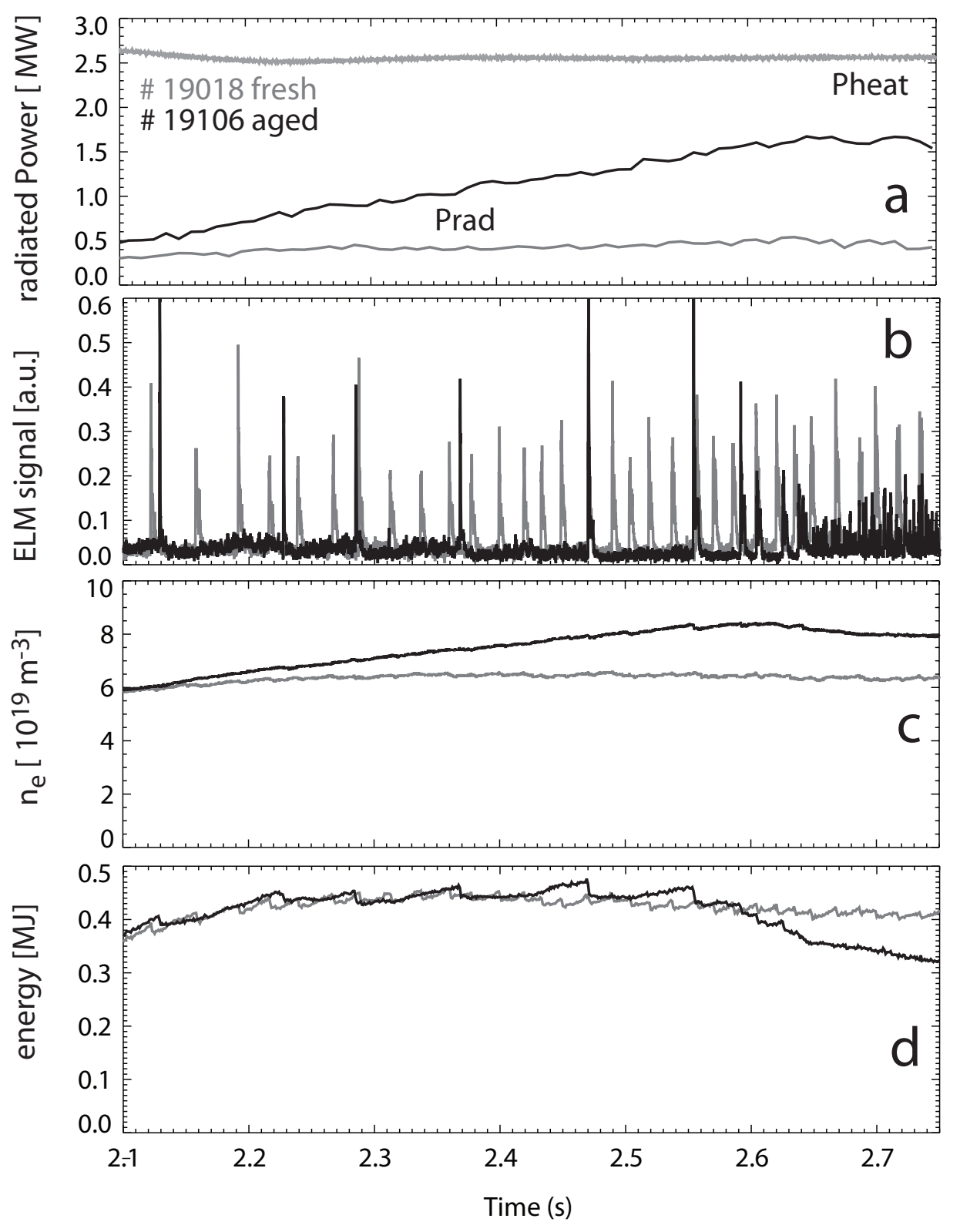

PSI17-P2-74

Fig 4 